\title{
Molecular regulation of the renin-angiotensin system by sodium-glucose cotransporter 2 inhibition in type 1 diabetes mellitus
}

\author{
Chantal Kopecky ${ }^{1,2}$ (D) - Yuliya Lytvyn ${ }^{3}$. Oliver Domenig ${ }^{4}$ - Marlies Antlanger ${ }^{2}$ - Johannes J. Kovarik ${ }^{2}$. \\ Christopher C. Kaltenecker ${ }^{2} \cdot$ Marko Poglitsch $^{4} \cdot$ Bruce A. Perkins $^{5} \cdot$ Kerry-Anne Rye $^{6} \cdot$ David Z. I. Cherney $^{3}$. \\ Marcus D. Säemann 7,8
}

Received: 14 September 2018 / Accepted: 20 March 2019 / Published online: 11 April 2019

(C) Springer-Verlag GmbH Germany, part of Springer Nature 2019

Keywords Angiotensins $\cdot$ Diabetes mellitus $\cdot$ Empagliflozin $\cdot$ Renin-angiotensin system activation $\cdot$ SGLT-2 inhibition

$\begin{array}{ll}\text { Abbreviations } \\ \text { Ang } & \text { Angiotensin } \\ \text { CKD } & \text { Chronic kidney disease } \\ \text { LLOQ } & \text { Lower limit of quantification } \\ \text { RAS } & \text { Renin-angiotensin system } \\ \text { SGLT-2 } & \text { Sodium-glucose cotransporter 2 } \\ \text { TGF } & \text { Tubuloglomerular feedback }\end{array}$

To the Editor: Sodium-glucose cotransporter-2 (SGLT-2) inhibitors are a highly promising class of glucose-lowering therapies and have been found to reduce cardiovascular and kidney disease risk in patients with type 2 diabetes [1-3]. The mechanisms underlying these cardio- and renoprotective effects of SGLT-2 inhibition are, however, incompletely understood and it is not known if these beneficial effects are also evident in people with type 1 diabetes.

David Z. I. Cherney and Marcus D. Säemann are joint senior authors.

Electronic supplementary material The online version of this article (https://doi.org/10.1007/s00125-019-4871-8) contains peer-reviewed but unedited supplementary material, which is available to authorised users.

Chantal Kopecky

c.kopecky@unsw.edu.au

1 School of Medical Sciences, Faculty of Medicine, UNSW Sydney, Level 2 Lowy Cancer Research Centre, High Street Gate 9, Sydney, NSW 2052, Australia

2 Department of Internal Medicine III, Division of Nephrology and Dialysis, Medical University of Vienna, Vienna, Austria

3 Department of Medicine, Division of Nephrology, Toronto General Hospital, University of Toronto, Toronto, ON, Canada
Hyperglycaemia triggers renal haemodynamic abnormalities in diabetes, which increases glomerular pressure and can lead to hyperfiltration and subsequent renal injury [4]. We have previously shown that empagliflozin increases distal tubular sodium delivery in people with type 1 diabetes, which enhances an autoregulatory mechanism in the kidneys (tubuloglomerular feedback [TGF]) that lowers glomerular pressure and attenuates hyperfiltration [5]. Although these renal haemodynamic effects in response to SGLT-2 inhibition resemble those of therapeutic renin-angiotensin system (RAS) blockade, TGF facilitates a distinct afferent vasoconstrictive mechanism instead of efferent vasodilatation as occurs with RAS blockade. It has therefore been hypothesised that SGLT-2 inhibition may promote global RAS activation resulting from composite renovascular effects such as volume depletion and osmotic diuresis [6].

4 Attoquant Diagnostics, Vienna, Austria

5 Mount Sinai Hospital, University of Toronto, Toronto, ON, Canada

6 School of Medical Sciences, Faculty of Medicine, UNSW Sydney, Sydney, NSW, Australia

7 6th Medical Department for Nephrology and Dialysis, Wilhelminenhospital, Vienna, Austria

8 Sigmund Freud Private University, Medical School, Vienna, Austria 
In this post hoc exploratory analysis of a prospective single-arm crossover intervention trial we investigated the effects of empagliflozin on RAS regulation to provide novel insights into physiological interactions between the RAS and SGLT-2 inhibition. We subjected available serum samples from 37 type 1 diabetes patients treated for 8 weeks with empagliflozin (25 mg once daily) (NCT01392560) [5] to simultaneous quantification of individual molecular RAS components. Steady-state angiotensin peptide levels in the serum samples were quantified by liquid chromatographytandem mass spectrometry (LC-MS/MS) [7], as described in detail in the electronic supplementary material (ESM) methods. None of the patients received any form of therapeutic RAS blockade. All participants provided informed consent following approval by the local ethics committee. Differences between the groups were compared using the Wilcoxon test. A $p$ value $<0.001$ was considered statistically significant.

Baseline characteristics of the study participants are shown in ESM Table 1. The patients had a mean $( \pm$ SD) age of $24 \pm$ 5 years, a mean diabetes duration of $16.6 \pm 7.2$ years and a mean baseline eGFR of $156 \pm 33 \mathrm{ml} \mathrm{min}^{-1}[1.73 \mathrm{~m}]^{-2}$ and $51 \%$ were male.

We found that 8 weeks of empagliflozin treatment resulted in a potent RAS activation, as indicated by increased median angiotensin (Ang) I and Ang II levels. The downstream metabolites, Ang III and Ang IV, were not significantly affected and remained largely below the lower limit of quantification (LLOQ, $<2.1$ and $<2.6 \mathrm{pmol} /$ 1) (Fig. 1a). Empagliflozin treatment increased RAS peptides Ang I and Ang II by twofold and 1.5-fold, respectively (Fig. 1b, c). Baseline levels of the alternative RAS effectors Ang (1-7) and Ang (1-5) were near or below the LLOQ in nearly all patients (Fig. 1d, e). Empagliflozin treatment increased Ang (1-5) in ten of the 37 patients, reflecting a conversion of Ang (1-7) into Ang (1-5). Plasma ACE activity was significantly decreased by empagliflozin (Fig. 1f), in contrast to a highly increased plasma renin activity (Fig. 1g). All measured levels of specific angiotensin peptides before and after empagliflozin treatment are summarised in ESM Table 2. We did not observe any significant correlations of changes in individual RAS components and changes in $\mathrm{HbA}_{1 \mathrm{c}}$ or sodium excretion but found a positive association of changes in Ang I and Ang II levels and diastolic blood pressure $(r=0.442, p=0.006$ and $r=0.183, p=0.034$, respectively).

We have previously shown that empagliflozin treatment activates intrarenal RAS components, including urinary angiotensinogen and ACE, and induces favourable ACE2 levels and enzyme activity in this type 1 diabetes cohort [8]. Our current observation that empagliflozin induces global RAS activation raises the possibility that combined
SGLT-2 and ACE inhibition may synergistically and favourably boost the alternative RAS axis. This intriguing hypothesis was followed up by ex vivo addition of a physiologically significant amount of ACE inhibitor (1 $\mu \mathrm{mol} / 1$ lisinopril) to samples from empagliflozintreated patients prior to RAS analysis, resulting in markedly elevated Ang (1-7) levels in $>25 \%$ of the samples, while significantly increasing Ang I and ablating Ang II and Ang (1-5) levels in the entire cohort (ESM Fig. 1).

Currently, RAS blocking agents are standard therapy for lowering blood pressure and reducing proteinuria in individuals with chronic kidney disease (CKD) [9]. Although RAS blockade has beneficial effects on kidney function, it cannot fully prevent CKD progression in many patients, particularly in the setting of diabetes. Our results imply that combined SGLT-2 and ACE inhibition result in favourable RAS profiles, which may further decrease the risk of kidney disease in diabetic patients. If activation of the alternative RAS is clinically important, it will also be essential to understand the relative contribution of specific RAS blockers to the cardiorenal protective effects of SGLT-2 inhibitors. A clinical study investigating the effects of combined SGLT-2 and ACE inhibition in CKD patients with and without type 2 diabetes mellitus is currently ongoing at our centre (NCT03078101), and the results are awaited with high interest.

Our current findings extend existing knowledge of SGLT-2 inhibitor-induced changes in renal physiology by demonstrating that empagliflozin specifically increases circulating angiotensin levels. Of note, the observed elevation in renin activity is in line with previous findings [5] and may explain why empagliflozin treatment increased Ang I to a greater extent than Ang II. Despite the physiological evidence of RAS upregulation, TGF activation leading to favourable afferent arteriolar constriction and consequently reduced glomerular hypertension is the dominant haemodynamic consequence of SGLT-2 inhibition, which may contribute to the beneficial clinical effects in the kidney.

Limitations of this study are the relatively small sample size and post hoc exploratory analysis of a prospectively conducted study. The results of our ex vivo analysis of the patient samples with the ACE inhibitor lisinopril only allow for a cautious and limited interpretation. Our data should therefore be viewed as explorative and hypothesis generating and require additional evaluation in vivo. Limitations of our RAS equilibrium analysis are that tissue or blood cell components do not contribute to the detected angiotensin levels and that these are solely dependent on the RAS enzyme profile present in the analysed samples. Moreover, the analyses were performed in a selected cohort of RAS blockade-naive type 1 diabetes patients with a mean age of 24 years without evidence of renal disease, and therefore cannot be generalised to a wider population. 
Fig. 1 Empagliflozin treatment changes angiotensin profiles. (a) Representative mass spectrometry-based RAS fingerprints of individuals with type 1 diabetes $(n=37)$ at baseline and after empagliflozin treatment. Results are presented as median of equilibrated and endogenous angiotensin levels (in pmol/l). Size of spheres and numbers aside represent absolute angiotensin concentrations ( $\mathrm{pmol} / \mathrm{l})$ analysed by mass spectrometry directly from patients' serum. Numbers [Ang I, II, III, IV, Ang (1-5), Ang (1-7)] signify specific angiotensin peptides, blue lines indicate RAS enzymes involved in respective angiotensin formation. AP, aminopeptidase A; NEP, neprilysin. (b-e) Serum levels of the classical RAS peptides Ang I (b) and Ang II (c) and the alternative RAS peptides Ang (1-7) (d) and Ang (1-5) (e) were measured at baseline (pre) and after (post) 8 weeks of empagliflozin treatment in type 1 diabetes patients $(n=37)$. LLOQ is given as values $<2.8 \mathrm{pmol} / 1$ for Ang (1-7) and $<3.0 \mathrm{pmol} / 1$ for Ang (1-5). (f) Plasma ACE activity surrogate (ACE-S) was calculated as the ratio of Ang II/I and (g) plasma renin activity surrogate (PRA-S) was calculated as sum of Ang I and Ang II. Results are presented as individual values and summarized as boxplots indicating the 25th and 75th percentiles with the line within the box marking the median. Whiskers above and below the box indicate the minimum and maximum values. $* * * p<0.001$ a

Pre treatment

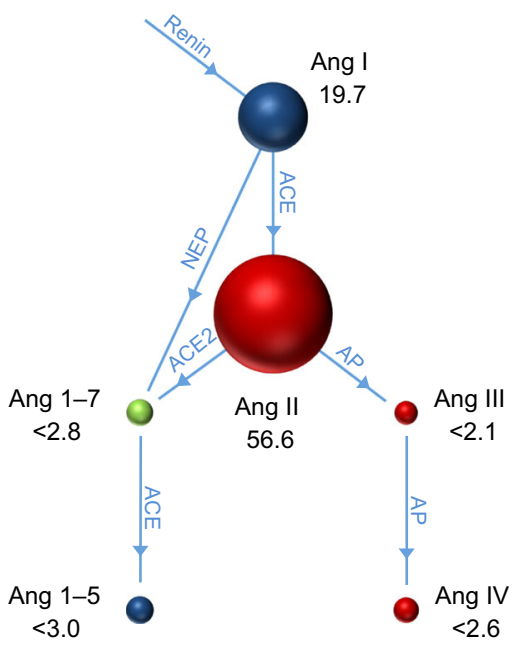

b

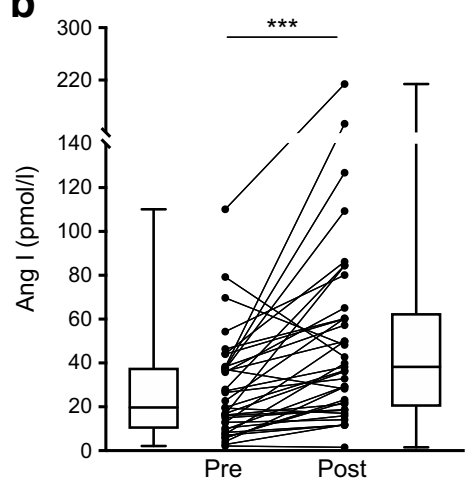

d

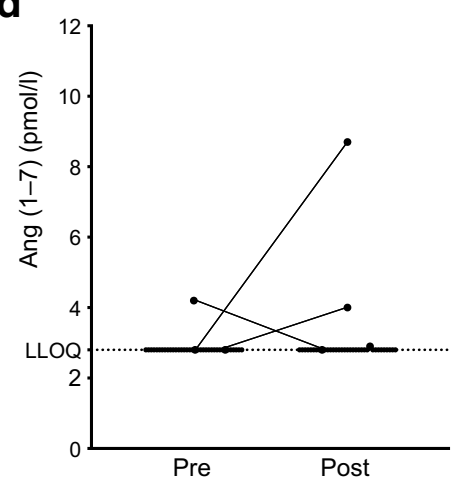

f

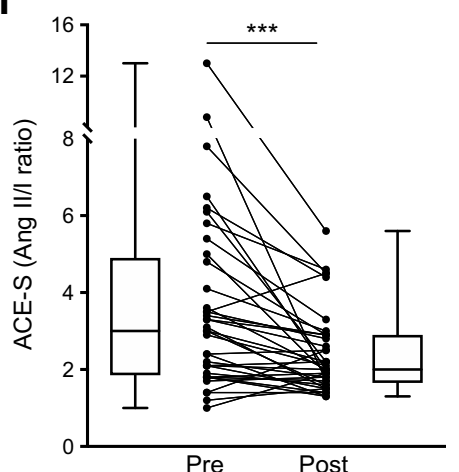

Post treatment

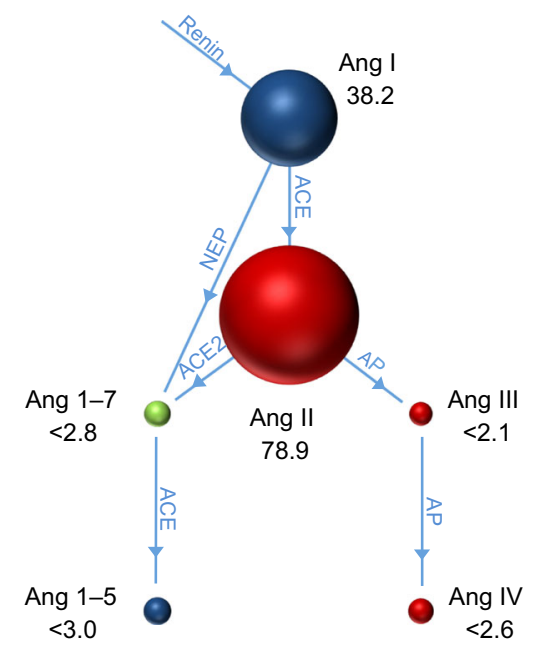

C

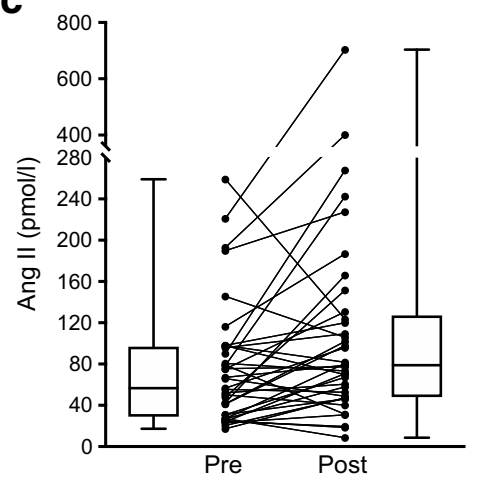

e
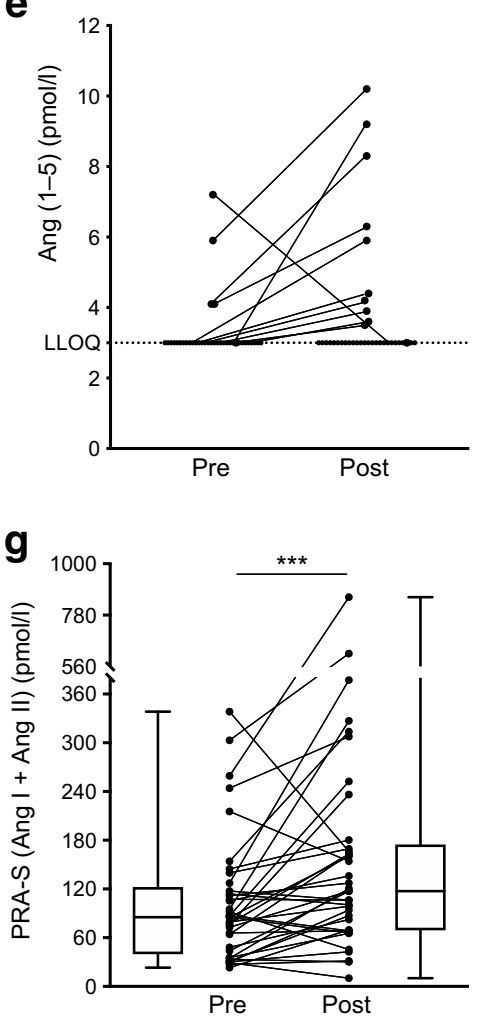
In conclusion, this study provides the first evidence of systemic RAS activation and modulation by the SGLT-2 inhibitor empagliflozin in type 1 diabetes. Our results also show that empagliflozin plus selective RAS blockade upregulates the key alternative RAS component Ang (17). These outcomes provide a compelling incentive for further studies of the nephroprotective effects of SGLT-2 inhibitors in larger populations. The clinical implications of a combined therapy may provide an opportunity to substantially improve current glucose-lowering, cardiovascular and renoprotective treatment options.

Acknowledgements Some of the data were presented as an abstract at the 45th ÖDG Annual Conference in 2017.

Data availability The authors declare that the data supporting the findings of this study are available within the article and its supplementary information files.

Funding DZIC is supported by funding from the Canadian Institutes of Health Research, the JDRF and the Banting and Best Diabetes Centre at the University of Toronto. DZIC is supported in part by a University of Toronto Merit Award, and his trainees are supported by the Canadian Diabetes Association (Diabetes Canada) Postdoctoral Fellowship, the University Health Network CaRE Fellowship Program. YL is supported by the Canadian Diabetes Association (Diabetes Care) Postdoctoral Fellowship. This research received no specific grant from any funding agency in the public, commercial or not-for-profit sector.

The study sponsor for the original study (NCT01392560) was not involved with the study design, analysis and interpretation of data, writing or decision to submit the work for publication for this present post hoc analysis.

Duality of interest DZIC has acted as a consultant and received honoraria from Boehringer Ingelheim, Lilly, Merck, Janssen, Sanofi, AbbVie, Mitsubishi-Tanabe Pharma and AstraZeneca and has received research operating funds from Boehringer Ingelheim-Lilly Diabetes Alliance, Merck, AstraZeneca and Janssen. BAP has received grants to his research institute and served on the advisory board for Boehringer Ingelheim. All other authors declare that they have no duality of interest associated with their contribution to this manuscript.

Contribution statement $\mathrm{CK}$ devised the study, analysed the data, wrote the manuscript and reviewed and edited the manuscript. DZIC and BAP conducted the original study, provided patient samples and contributed to data interpretation. MP and OD performed mass spectrometry analyses and YL acquired epidemiological data of the patient cohort and performed correlation analysis. All named authors contributed to data interpretation, discussion, helped revise the manuscript providing critical intellectual content and approved it for publication. CK is the guarantor and takes full responsibility of the work as a whole, including the study design, access to data and the decision to submit and publish the manuscript.

\section{References}

1. Zinman B, Wanner C, Lachin JM et al (2015) Empagliflozin, cardiovascular outcomes, and mortality in type 2 diabetes. N Engl J Med 373(22):2117-2128. https://doi.org/10.1056/NEJMoa1504720

2. Neal B, Perkovic V, Mahaffey KW et al (2017) Canagliflozin and cardiovascular and renal events in type 2 diabetes. N Engl J Med 377(7):644-657. https://doi.org/10.1056/NEJMoa1611925

3. Wanner C, Inzucchi SE, Lachin JM et al (2016) Empagliflozin and progression of kidney disease in type 2 diabetes. $\mathrm{N}$ Engl $\mathrm{J}$ Med 375(4):323-334. https://doi.org/10.1056/NEJMoa1515920

4. Reidy K, Kang HM, Hostetter T, Susztak K (2014) Molecular mechanisms of diabetic kidney disease. J Clin Invest 124(6):2333-2340. https://doi.org/10.1172/JCI72271

5. Cherney DZ, Perkins BA, Soleymanlou N et al (2014) Renal hemodynamic effect of sodium-glucose cotransporter 2 inhibition in patients with type 1 diabetes mellitus. Circulation 129(5):587-597. https://doi.org/10.1161/CIRCULATIONAHA.113.005081

6. Heerspink HJ, Perkins BA, Fitchett DH, Husain M, Cherney DZ (2016) Sodium glucose cotransporter 2 inhibitors in the treatment of diabetes mellitus: cardiovascular and kidney effects, potential mechanisms, and clinical applications. Circulation 134(10):752772. https://doi.org/10.1161/CIRCULATIONAHA.116.021887

7. Roksnoer LC, van Veghel R, de Vries R et al (2015) Optimum AT1 receptor-neprilysin inhibition has superior cardioprotective effects compared with AT1 receptor blockade alone in hypertensive rats. Kidney Int 88(1):109-120. https://doi.org/10.1038/ki.2015.107

8. Cherney DZ, Perkins BA, Soleymanlou N et al (2014) Sodium glucose cotransport-2 inhibition and intrarenal ras activity in people with type 1 diabetes. Kidney Int 86(5):1057-1058. https://doi.org/10. 1038/ki.2014.246

9. Klein WS, Merz CN, Jenkins MR (2014) Guidelines for managing high blood pressure. JAMA 312(3):294-295. https://doi.org/10. 1001/jama.2014.6593

Publisher's note Springer Nature remains neutral with regard to jurisdictional claims in published maps and institutional affiliations. 